\title{
Educação em saúde ao ostomizado: um estudo bibliométrico
}

\author{
HEALTH EDUCATION TO OSTOMY PATIENTS: A BIBLIOMETRICS STUDY
}

ORIENTACIÓN ALA SALUD DEL OSTOMATO: UN ESTUDIO DE BIBLIOMETRÍA

\author{
Audrey Garcia Reveles ${ }^{1}$, Regina Toshie Takahashi²
}

\section{RESUMO}

Este trabalho objetivou identificar a produção científica sobre orientação ao ostomizado, publicada entre 1970 e 2004 e classificá-la segundo quantidade, cronologia de publicação, função exercida pelos autores, procedência, tipo, assunto, origem e palavras-chave; utilizando a metodologia bibliométrica. As 27 publicações foram coletadas no banco de dados DEDALUS, nas bases de dados LILACS, MEDLINE e de uma docente da EEUSP (referência nacional em ostomia). No total, 19 são nacionais, oito internacionais e escritas principalmente por enfermeiros e estomaterapeutas. Os tipos são: dissertações, teses, folhetos, livros e artigos. A procedência dos materiais é da academia, laboratorial e hospitalar. A década de noventa é a que concentra o maior número de trabalhos nesta área temática. Todos têm como finalidade elevar a auto-estima dos pacientes para fazê-los sentir que, mesmo com uma ostomia, eles podem levar uma vida normal. Assim, o estudo mostrou que a enfermeira como educadora de um ostomizado deve conhecer tais publicações para melhorar a assistência.

\section{ABSTRACT}

This study has as its objective to identify the scientific studies dealing with the orientation to patients submitted to ostomy published between 1970 to 2004 and to classify them according to quantity, chronology of publication, authors' function, source, kind of study, topic, origin and key words by using the bibliometrics methodology. A total of 27 publications were collected in the DEDALUS databank, in the LILACS and MEDLINE databases and from a professor of the University of São Paulo's Nursing School (EEUSP, which is a national reference in ostomy in Brazil). Of these, 19 were written by Brazilians and 8 by nonBrazilians; most were written by nurses and enterostomal therapists. Dissertations, theses, orientation manuals, books and articles were found. The origin of the material was academia, laboratories and hospitals. The 1990s concentrated the largest number of studies in this thematic area. All of them have the purpose of elevating patients' self-esteem in order to make them feel that, even with an ostomy, they can have a normal life. Thus the study concludes that the nurse, as an educator for the ostomy patient, should be acquainted with those publications to improve the assistance he/she provides.

\section{RESUMEN}

Este trabajo tuvo como objetivo identificar la producción científica acerca de la orientación al ostomato, publicada entre $1970 \mathrm{y}$ 2004 y clasificarla según la cantidad, cronología de la publicación, función de los autores, fuente, clase, asunto, origen y palabras-clave; utilizando la metodología de bibliometría. Las 27 publicaciones se encuentran en la base de datos DEDALUS, LILACS, MEDLINE y en la base de datos de un profesor de la EEUSP (referencia nacional en ostomía). En suma, 19 son nacionales y 8 internacionales; están escritas principalmente por enfermeros y terapeutas enterostomales. Las referencias son: disertaciones, teorías, prospectos, libros y artículos. Los materiales son de la academia, del laboratorio y del hospital. La década de los noventa concentró el mayor número de trabajos en esta área temática. Todos tienen como propósito elevar la autoestima de los pacientes para que sientan que, aún con ostomía, pueden llevar una vida normal. Así, concluimos que el enfermero, como educador de un ostomato debe conocer estas publicaciones para una mejor ayuda.

\author{
1 Graduanda da \\ Escola de \\ Enfermagem da \\ Universidade de São \\ Paulo-EEUSP. \\ audreyreveles@ \\ bol.com.br \\ 2 Professora Doutora \\ do Departamento de \\ Orientação \\ Profissional da \\ Escola de \\ Enfermagem da \\ Universidade de São \\ Paulo-EEUSP. \\ rttakaha@usp.br
}

$\begin{array}{lll}\text { DESCRITORES } & \text { KEY WORDS } & \text { DESCRIPTORES } \\ \text { Educação em saúde. } & \text { Health education. } & \text { Educación en salud. } \\ \text { Ostomia. } & \text { Ostomy. } & \text { Ostomía. } \\ \text { Enfermagem. } & \text { Nursing. } & \text { Enfermería. }\end{array}$




\section{INTRODUÇÃO}

Na Enfermagem a educação em saúde é um instrumento fundamental para uma assistência de boa qualidade, pois o enfermeiro além de ser um cuidador é um educador, tanto para o paciente quanto para a família, realizando orientações.

Neste trabalho, entendemos educação em saúde como um processo de ensino que o enfermeiro faz com seus clientes com o objetivo deles aprenderem a se autocuidarem, além de se tornarem multiplicadores dos conhecimentos da área de saúde.

Nesta perspectiva de orientação ao paciente para o seu autocuidado foi desenvolvido este trabalho, o qual enfocou a educação em saúde voltada para adultos.

Existem muitas teorias de Educação que definem o processo educação em saúde, umas mais rígidas, outras mais flexíveis, porém para as autoras, a educação em saúde deve considerar que todos os elementos envolvidos são importantes, tanto os profissionais da área quanto os pacientes, e que devem participar de forma ativa na construção do conhecimento.

Assim, neste trabalho focamos a educação em saúde voltada para o paciente adulto ostomizado.

A ostomia é

um procedimento cirúrgico, no qual é realizada uma abertura artificial entre o intestino e a parede abdominal: o ostoma é o local através do qual se dá a passagem do conteúdo intestinal(1).

O processo ensino-aprendizagem do adulto ostomizado começa no pré-operatório, no qual a enfermeira deve estabelecer um bom vínculo com o paciente e a família para ajudá-los a começar a compreender como é a situação concreta a fim de melhor se adaptarem à mudança do estilo de vida.

Em seguida, no pós-operatório tem início uma abordagem técnica relacionada ao autocuidado do paciente, que se refere em como proteger a pele ao redor do ostoma, como trocar a bolsa de ostomia, fazer a higiene do ostoma, como se alimentar e evitar a formação de gases.

Por fim, a aprendizagem continua no domicílio e em grupos de apoio com a finalidade que o paciente e sua família encontrem maneiras de viver normalmente, mesmo tendo que conviver com uma ostomia.

\section{OBJETIVOS}

- Identificar a produção científica nacional e interna- cional sobre a orientação ao paciente ostomizado, publicada no período entre 1970 a 2004;

- Classificar a produção científica encontrada segundo quantidade, cronologia de publicação, função exercida pelos autores, procedência, tipo, assunto, origem e palavras-chave dos trabalhos.

\section{MÉTODO}

Esse estudo foi desenvolvido na perspectiva de uma pesquisa bibliométrica.

"O termo bibliometria é utilizado para quantificar os processos de comunicação escrita e, o emprego de indicadores bibliométricos para medir a produção científica" ${ }^{\text {"(2) }}$. Justifica-se a sua utilização pelos seguintes motivos:

- A análise e avaliação das fontes difusoras dos trabalhos;

- A evolução cronológica da produção científica;

- A produtividade de autores e instituições;

- A propagação das publicações científicas;

- O crescimento de qualquer campo da ciência;

- O envelhecimento dos campos científicos;

- O impacto das publicações frente à comunidade científica internacional.

Para análise das publicações catalogadas fundamentouse nas abordagens de pesquisa qualitativa e quantitativa, "as quais são perspectivas complementares quando se pretende aproximar de uma realidade com a finalidade de conhecê-la"(3).

Em termos qualitativos foram analisados os assuntos tratados nas publicações pesquisadas, quanto ao conteúdo, ordem dos temas, ilustrações, linguagem objetiva e acessível.

Quanto aos dados quantitativos procurou-se investigar as seguintes variáveis: o banco ou base de dados onde foram extraídos os trabalhos, os autores, as atividades exercidas pelos autores, as palavras-chave, a origem, o tipo e a procedência dos materiais e $o$ ano de publicação.

\section{Etapas para coleta de dados}

Primeiro consultamos o banco de dados DEDALUS e as bases de dados: BDENF, MEDLINE E LILACS com os seguintes descritores: processo ensino-aprendizagem em saúde; educação do paciente; orientação à saúde e enfermagem.

Depois, contatamos uma docente que é considerada referência em ostomia, da Escola de Enfermagem da Universidade de São Paulo (EEUSP) que nos cedeu as demais referências.

Então criamos uma ficha de catalogação para coletar os 
dados, com os seguintes itens: banco de dados, palavra-chave, autor, atividade exercida pelo autor, origem do material, referencial teórico, ano, tipo, procedência do material e assunto.

Desta maneira, após a leitura e fichamento das publicações, separou-se as idéias centrais em categorias dos limites e vantagens cujos elementos relacionam-se entre $\mathbf{s i}^{(3)}$.

\section{RESULTADOS E ANÁLISE}

O referencial bibliográfico encontrado passou por um processo de distribuição por ano de publicação, função exercida pelos autores, procedência do material (da academia, laboratorial, de uma associação ou hospitalar), tipo de publicação (artigo, dissertação, tese, livro ou manual de orientação), origem (país), palavras-chave dos trabalhos e por categorias a serem identificadas por resumo da obra ${ }^{(1)}$.

Considerando-se os objetivos deste artigo, baseados na pesquisa bibliométrica, "a análise dos dados obtidos teve a finalidade de buscar os seguintes resultados"(1):

- Quantas publicações foram produzidas no período em análise;

- Qual é a função exercida pelos autores;

- Qual a procedência do material: da academia, de um laboratório, de uma associação ou de um hospital;

- Quais os tipos de publicação da produção científica;

- Quais são as palavras-chave dos trabalhos;

Como é a linguagem, a ilustração e a escrita que norteiam a produção científica.
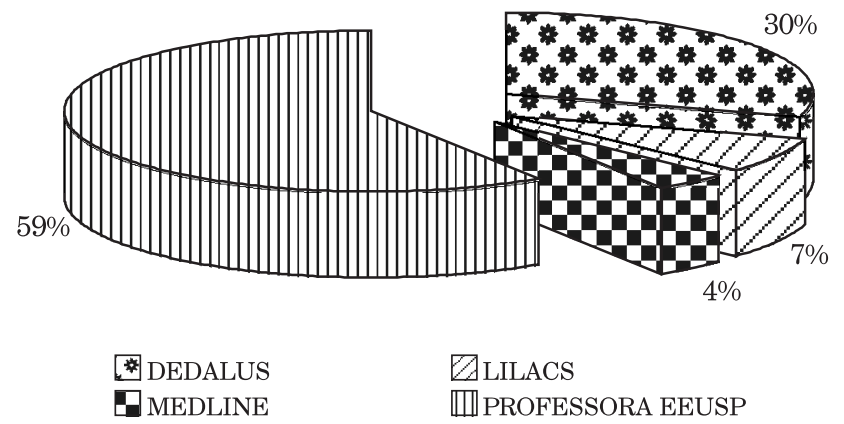

Figura 1 - Distribuição da Produção Científica sobre a Educação em Saúde dos Adultos Ostomizados referente à localização na Base de Dados - São Paulo - 2004
Conforme mostra a Figura 1, foram identificadas 27 publicações, sendo que destas $8(29,6 \%)$ estão no banco de dados DEDALUS, $2(7,4 \%)$ na base de dados LILACS, $1(3,7 \%)$ na base de dados MEDLINE e 16 $(59,2 \%)$ na base de dados da professora referência em ostomia da EEUSP. Há um predomínio de publicações no material bibliográfico da professora, pois esta base é composta apenas de trabalhos referentes à ostomia.

Das 27 publicações estudadas, $18(66,6 \%)$ não possuem palavras-chave e $9(33,3 \%)$ possuem, as quais são: reabilitação, ostomia, cuidados de enfermagem, autocuidado, educação do paciente, cuidados com a colostomia, irrigação, pacientes ostomizados, preocupações, percepções, problemas, psicologia, relações interpessoais, educação em saúde, grupos de auto-ajuda, acontecimentos que mudam a vida, processo ensino-aprendizagem em saúde, colostomia, ileostomia, urostomia. As palavras-chave mais utilizadas foram: ostomia, cuidados de enfermagem, autocuidado e educação do paciente.

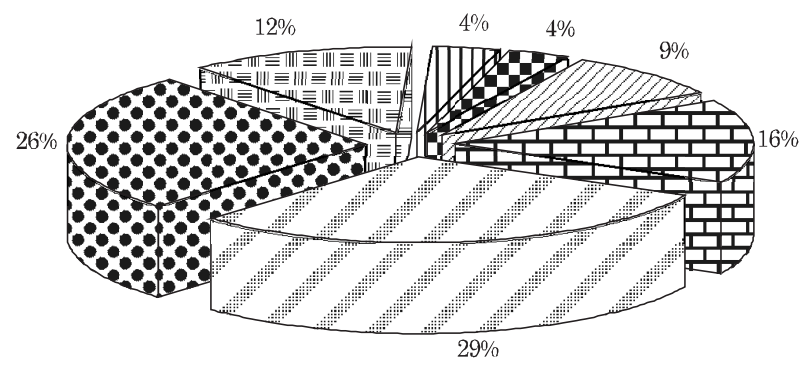

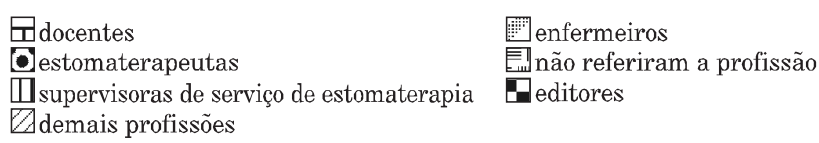

Figura 2 - Distribuição da Produção Científica sobre a Educação em Saúde dos Adultos Ostomizados referente às atividades que os autores exercem - São Paulo - 2004

Dos 27 trabalhos analisados verificou-se 54 autores com uma média de $2(3,7 \%)$ autores por trabalho. Quanto às atividades que esses autores exercem, consta-se que $9(16,6 \%)$ são docentes, $17(31,5 \%)$ são enfermeiros, $15(27,7 \%)$ são estomaterapeutas, $7(13 \%)$ não referiram a profissão, $2(3,7 \%)$ supervisoras de serviço de estomaterapia, $2(3,7 \%)$ editores, $5(9,2 \%)$ demais profissões, tais como: auxiliar e técnico de Enfermagem, nutricionista, cirurgião, presidente de uma associação de ostomizados.

Estes dados mostram que a área de ostomia é um bom campo para a enfermagem, pois esta profissão é bem preparada para trabalhar tanto a assistência quanto a educação do paciente, as quais são as ferramentas fundamentais para cuidar de um ostomizado. 
Em relação à origem do material estudado, 19 $(70,3 \%)$ são nacionais e $8(29,6 \%)$ são internacionais dos seguintes países: Dinamarca, Portugal, Austrália, Nova Zelândia, Espanha e França.

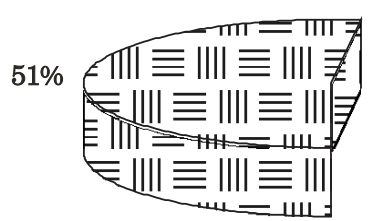

피 década de 70

罡 década de 90

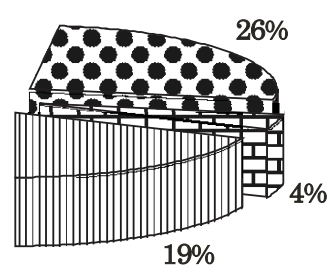

IIII década de 80

2000 a 2004
Figura 3 - Distribuição da Produção Científica sobre a Educação em Saúde dos Adultos Ostomizados referente ao período entre 1971 a 2004 por Décadas - São Paulo - 2004

O período submetido à análise bibliométrica está compreendido entre o ano de 1971 até o ano de 2004, sendo que este período foi dividido em décadas para apresentação dos dados. Como pode ser observado na figura 3 há apenas 1 $(3,7 \%)$ trabalho da década de $70,5(18,5 \%)$ da década de oitenta, $14(51,8 \%)$ da década de noventa e $7(25,9 \%)$ do ano 2000 a 2004. Assim a década de noventa é a que possui a maior concentração de trabalhos neste estudo bibliométrico.

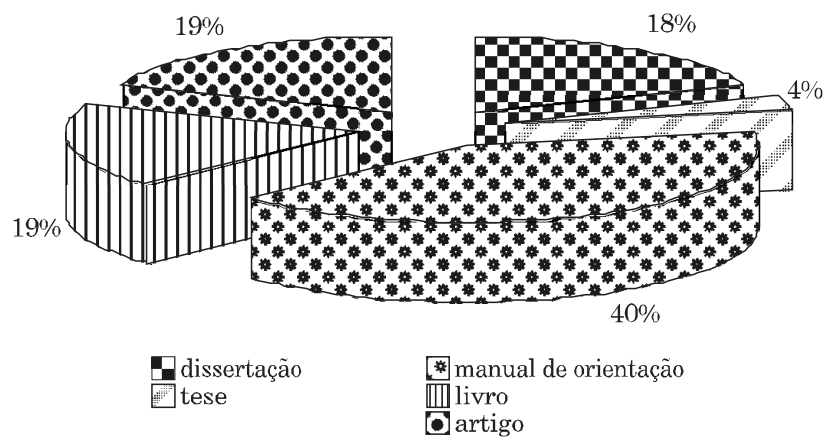

Figura 4 - Distribuição da Produção Científica Nacional e Internacional da Educação em Saúde dos Adultos Ostomizados referente ao Tipo de Referência - São Paulo - 2004

Os trabalhos que compõem este estudo são dos seguintes tipos: dissertação $(18,5 \%)$, tese $(3,7 \%)$, manual de orientação $(40,7 \%)$, livro (18,5\%), artigo $(18,5 \%)$, sendo que dos artigos, 2 são da Revista Brasileira de Enfermagem, 1 da Revista da Escola de Enfermagem da USP, 1 da Revista Gaúcha de Enfermagem e 1 do Journal Wound Ostomy Continence Nursing. O fato de haver mais manuais de orientação pode estar relacionado ao patrocínio dos grandes laboratórios que produzem materiais utilizados pelos ostomizados.

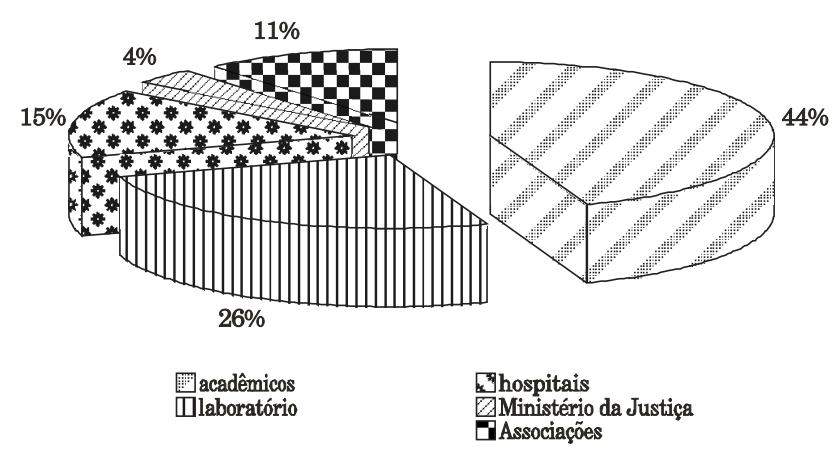

Figura 5 - Distribuição da Produção Científica sobre a Educação em Saúde dos Adultos Ostomizados referente à procedência do material - São Paulo - 2004

Na figura 5 pode ser observado que em relação à procedência do material, $12(44,4 \%)$ são da academia, $7(25,9 \%)$ são de laboratórios, 4 (14,8\%) são de hospitais, 1 (3,7\%) é do Ministério da Justiça, $3(11,1 \%)$ são de associações de enfermeiros e ostomizados. Os materiais de laboratórios são procedentes de empresas nacionais e internacionais e os provenientes de hospitais são de instituições privadas de grande porte da cidade de São Paulo.

Após a apresentação dos dados da figura acima podemos ressaltar que a maioria dos materiais tem procedência da academia, talvez porque os autores estejam ligados à cursos de pós-graduação e assim são "aqueles que normalmente apresentam maior proximidade com as atividades de pesquisa. No entanto, trata-se de uma lacuna importante na enfermagem brasileira a falta do enfermeiro assistencial, já que tem maior contato com a problemática emergente da prática"(3).

Todas as publicações deste trabalho seguem uma mesma abordagem, o qual consiste nos seguintes tópicos:

1. Anatomia e fisiologia do aparelho digestório: com ilustrações da boca, faringe, esôfago, estômago, intestino delgado (duodeno, jejuno e íleo), intestino grosso (ceco, colon ascendente, colon transverso, colon descente, colon sigmóide, reto e ânus), explicação sobre as etapas da digestão (ingestão, mastigação, deglutição, digestão, absorção e excreção).

2. Anatomia e fisiologia do aparelho urinário: com ilustrações dos rins, ureteres, bexiga e uretra, explanação sobre as funções renais, tais como: regulação do equilíbrio ácidobásico e hidroeletrolítico, função endócrina, produção de aldosterona pela supra-renal e a formação de urina.

3. O que é uma ostomia: é uma abertura cirúrgica realizada na parede abdominal, onde uma porção do intestino é levada até a pele, no caso de uma ostomia digestiva, para a drenagem de fezes ou para drenagem de urina no caso de uma ostomia urinária. 
4. Tipos de ostomas: urostomia, ileostomia, colostomia ascendente, colostomia transversa, colostomia descendente/sigmoidostomia.

5. Como cuidar do ostoma: higiene com água e sabão e troca da bolsa sendo que a freqüência de esvaziamento depende do tipo de ostomia.

6. Como conviver com um ostoma: trabalho, lazer, esportes, sexualidade, alimentação, relacionamentos familiares, vestuário e viagens.

\section{Grupos de apoio emocional e financeiro.}

Constatamos que os tópicos são apresentados exatamente na mesma ordem citada e de forma bem simplificada, muito ilustrativa, mostrando ao ostomizado que ele terá uma nova vida e que mesmo com a ostomia, ela poderá ser normal.

Esta técnica de utilização de materiais complementares como objetivo de reforçar as informações verbais "tem sido descrita por educadores como a melhor forma de ensinar indivíduos adultos, visto que as pessoas retêm apenas $20 \%$ do que ouvem, $30 \%$ do que vêem, $50 \%$ do que ouvem e vêem, $70 \%$ do que ouvem, vêem e dizem e $90 \%$ do que ouvem, vêem, dizem e fazem"(4).

Esta padronização de orientação ao paciente ostomizado assegura que ele terá apoio em qualquer lugar do mundo para tirar dúvidas ou esclarecer problemas e também facilita o trabalho dos profissionais de saúde através de uma comunicação clara e objetiva que resulta numa melhor assistência ao paciente. Porém, "estas informações padronizadas podem não corresponder às necessidades de cada paciente"(4).

Os itens que diferem em alguns trabalhos são: complicações da colostomia, interação entre enfermeiro e ostomizado, auto-irrigação por colostomizados, aspecto emocional dos ostomizados, visita domiciliária para ostomizado, despreparo da equipe de enfermagem para cuidar de ostomias, adolescente ostomizado, orientação à mãe com uma criança ostomizada, doenças associadas a colostomia, aspectos históricos de cuidado com o ostoma, gravidez com uma ostomia, medicação e casamento.

\section{REFERÊNCIAS}

1. Benedini Z. Pesquisando para educar: a "Pedagogia do Ostomizado" e a prática da visita domiciliária [tese]. Ribeirão Preto: Escola de Enfermagem de Ribeirão Preto, Universidade de São Paulo; 1993.

2. Oliveira JC. Estudo bibliométrico das publicações de custos em enfermagem no período de 1966 a 2000 [dissertação]. São Paulo: Escola de Enfermagem, Universidade de São Paulo; 2001.

3. Martin MJS, Messias DCC, Ostroski LE. Análise das publicações sobre diagnóstico de enfermagem no Brasil. Nursing (São Paulo). 2004;76(7):23-8.

\section{CONSIDERAÇÕES FINAIS}

Do levantamento das publicações sobre a educação em saúde do adulto ostomizado, pode-se concluir que o grande desafio dos enfermeiros é melhorar a qualidade da assistência através da implementação de instrumentos para proporcionar ao cliente um cuidado mais humanizado.

Este trabalho, um estudo bibliométrico sobre orientação ao ostomizado, é uma fonte de informação que poderá auxiliar os enfermeiros para aumentar as informações e consequentemente melhorar a assistência na área de ostomia, pois ele nos mostra que a orientação é um importante instrumento e que a capacitação de recursos humanos especializados é essencial ${ }^{(5)}$.

Todas as publicações sobre as orientações aos pacientes ostomizados têm como objetivo esclarecer as dúvidas do paciente e de sues familiares e principalmente elevar a auto-estima do paciente, com enfoque nítido nos manuais de orientação ao ostomizado, utilizando adequada apresentação e com uma linguagem acessível.

A maior concentração das publicações analisadas nesta pesquisa situou-se na década de noventa.

Outro aspecto importante observado nas publicações analisadas foi a padronização da linguagem, a qual facilita o trabalho do enfermeiro, pois este sabe que o seu paciente será orientado em um mesmo referencial independente do lugar que esteja.

Também observamos na nossa prática que apesar do grande número de publicações referentes ao assunto ostomia, muitos enfermeiros ainda se encontram despreparados para assistir aos ostomizados, desta forma uma outra pesquisa para trabalhar esta problemática pode ser realizada.

Com isso vemos a necessidade de uma educação continuada aos enfermeiros que trabalham no setor de ostomias para que cada hospital organize grupos de enfermeiros estomaterapeutas e possam desenvolver seus próprios manuais de acordo com as características e necessidades de seus clientes ostomizados.

4. Ducci AJ, Pimenta CAM. Programas educativos e a dor oncológica: estudo de revisão. Rev Bras Cancerol. 2003;49(3):185-92.

5. Diogo MJDE, Ceolim MF, Cintra FA. Orientações para idosas que cuidam de idosos no domicílio. Rev Esc Enferm USP. 2005;39(1):97-102. 


\section{Produção científica levantada}

Benedini Z. Pesquisando para educar: a "Pedagogia do Ostomizado" e a prática da visita domicilária [tese]. Ribeirão Preto: Escola de Enfermagem de Ribeirão Preto, Universidade de São Paulo; 1993.

Borges EL, Nunes AA. Como cuidar da criança ostomizada. Belo Horizonte: Ed.UFMG; 1999. (Coleção: Quem sabe faz saúde n. 21).

Borges LLN. Manual de ostomia. Salvador: Hospital Aliança; 2001.

Bradan A, Bérard M, Bernard D, Berta JL, Bocquet M, Boisse $\mathrm{Y}$, et al. Aujourd'hui, la vie recommence. $6^{\mathrm{a}}$ ed. Paris (FR): Laboratoires Convatec; 1991.

Carvalheira C, organizadora. Ainda posso levar uma Vida Normal? Brasília: Ministério da Justiça/Secretaria de Estado dos Direitos Humanos/ CORDE; 1999.

Casas GV. Guia del ostomizado digestivo: cuidados de enfermeria em la atencion primaria. Madrid (ES): Laboratório Coloplast; 1990.

Cezareti IUR. Ostomizado: reabilitação sem fronteiras? Ponto de vista do enfermeiro. Rev Bras Enferm. 1995;48(1):60-5.

CHADU. Iseo and the ostomaties. Denmark: Medical Strip Cartoon Collection; 1992.

Chirinos NEC. Modelo conceitual e operacional de educação para o autocuidado de indivíduos colostomizados [dissertação]. Florianópolis: Universidade Federal de Santa Catarina; 1988.

Fernandes I, organizador. Ostomizado: redescobrindo a vida saudável. Porto Alegre: Associação Gaúcha de Ostomizados; 2001.

Ferreira AC, Seiça A, Mineiro C, Neves D, Pereira H, Silva I. et al. Viver e ser independente. Coimbra (PO): Laboratório: Coloplast; 2003.

Hawley J. Living with your ostomy. Christchurch (NZ): Federation of N. Z. Ostomy Societies; 1997.

Hughes ESR, Cuthbertson AM, Hardy KJ. Stoma \& fistula management: a practical guide. Melbourne (AU): E. R. Squibb \& Sons Pty; 1981.

Mantovani MF. O processo de interação propiciando ensino e aprendizagem na vivência com a ostomia [dissertação]. Florianópolis: Universidade Federal de Santa Catarina; 1996.
Marcondes MG. Cuidando de sua ostomia. São Paulo: Laboratório Convatec; 2003.

Marcondes MG. Manual de ostomia. São Paulo: Laboratório Convatec; 2004.

Martelli ZB. Necessidades de aprendizagem da pessoa colostomizada: impressão diagnóstica do enfermeiro através da observação limitante e taxonomia de objetivos educacionais [dissertação]. Ribeirão Preto: Escola de Enfermagem de Ribeirão Preto, Universidade de São Paulo; 1983.

Martins ML. Ensinando e aprendendo, em grupo, a enfrentar situações vivenciadas por pessoas ostomizadas. [dissertação]. Florianópolis: Universidade Federal de Santa Catarina; 1995

O'Shea HS. Teaching the adult ostomy patient. J Wound Ostomy Continence Nurs. 2001;28(1):47-54.

Oliveira C. Assistência de enfermagem aos pacientes colostomizados. Rev Bras Enferm.1971;24(6):107-17.

Pires RP, Moritaka LT, Piseta V, Onofre A. Colostomizado e ileostomizado: guia de orientação de enfermagem. São Paulo: Hospital Alemão Oswaldo Cruz; 1999.

Pires RP, Pisetta V, Moritaka LT, Onofre A. Irrigação de colostomia: manual de orientação de enfermagem. São Paulo: Hospital Alemão Oswaldo Cruz; 1999.

Santos VLCG, Kozumi MS. Estudo sobre os resultados da irrigação em colostomizados submetidos a um processo de treinamento sistematizado. Rev Esc Enferm USP. 1992;26(3):303-14.

Santos VLCG. Estudo sobre os resultados da irrigação em colostomizados submetidos a um processo de treinamento sistematizado [dissertação]. São Paulo: Escola de Enfermagem, Universidade de São Paulo; 1989.

Senna PA, Rodrigues VC, Almeida RS. Cuidados com sua jejunostomia. São Paulo: Hospital do Câncer; 2000.

Stoma Care Nurses Interest Section (NZNA). For the person with a stoma: towards a normal life. Wellington (NZ); 1988.

Trentini M, Pacheco MAB, Martins ML, Silva DMGV, Farias SR, Duarte R, et al. Vivendo com um ostoma: um estudo prelimiar. Rev Gaúcha Enferm. 1992;13(2):22-8. 